\title{
Can a Quantum Electron Microscope Achieve Low-Damage (Biological) Imaging?
}

\author{
Sebastian Thomas ${ }^{1,2}$, Jakob Hammer ${ }^{1,2}$, Philipp Weber ${ }^{1}$ and Peter Hommelhoff ${ }^{1,2}$ \\ 1. Friedrich-Alexander-Universität Erlangen-Nürnberg, Department Physik, Erlangen, Germany \\ 2. Max-Planck-Institut für Quantenoptik, Garching, Germany
}

Recently, a new type of electron microscope has been proposed which is based on the exploitation of quantum effects in order to reduce sample damage [1]. The proposed scheme potentially allows imaging with arbitrarily low radiation damage, which is promising for any application where radiation damage is particularly problematic. One example is the imaging of biological samples in an electron microscope.

The quantum mechanical process behind the new electron microscopy scheme is known as an "interaction-free measurement" (IFM) and has been studied both theoretically and experimentally with photons as probe particles instead of electrons [2-5]. While classical imaging is based on detecting the presence of a sample via the scattering or absorption of probe particles like electrons or photons (with any interaction between probe particle and sample potentially causing damage), interaction-free measurements can detect the presence of a sample without direct interaction between probe particle and sample and hence vanishing damage. This is accomplished by putting the imaging system into an interferometric setup. Examples for both photons and electrons as probe particles are shown in Figure 1.

The IFM scheme is related to the quantum Zeno effect [6] and is best explained on the single-particle level. Passing through a beam splitter, a quantum particle's wavefunction is split into two parts with unequal amplitudes that are spatially separated but remain coherent with each other. The part with the majority of the amplitude is called the reference beam and the part with only a small part of the amplitude is called the sample beam. While the path of the reference beam is free, the path of the sample beam may or may not contain an opaque sample to be imaged. If there is a sample in the beam, its presence causes the two parts of the wavefunction to decohere, triggering a position measurement (in which beam the particle is to be found). As the amplitude in the sample beam is much smaller than the amplitude in the reference beam, this will most likely lead to the particle being localized in the reference beam - representing the quintessential quantum mechanical wavefunction collapse associated with a position measurement, here induced by the sample. Subsequently the two beams pass through a beam splitter again, which causes a transfer of amplitude between them. This process is repeated $N$ times until the amplitude is fully transferred into the sample beam in the case where there is no sample present $(N$ depending on the reflectivity of the beam splitter). The resulting amplitudes are shown in Figure 2.

Finally, a detector is used to determine whether the particle is in the reference beam or the sample beam after $N$ round trips through the setup. If there is no sample, the amplitude will be completely transferred to the sample beam and the particle can be detected there. If there is a sample blocking the sample beam, the particle will most likely be found in the reference beam. Crucially, detecting the particle in the reference beam implies the presence of the sample without any damaging interaction between particle and sample having occurred. This is why this measurement scheme is called "interaction-free". Additionally, the particle can be lost during the process due to the sample interaction, which means that the IFM was unsuccessful. By increasing $N$ (via a change of the beam splitter's reflectivity), the probability of losing the particle and, hence, inducing damage can be made arbitrarily low [3]. 
While there are many possible ways of implementing an IFM, they always rely on multiple coherent splittings of a quantum particle's wavefunction. For this reason, the biggest technical challenge in the realization of such a scheme in an electron microscope is the development of a suitable beam splitter (like the double-well potential proposed in Ref. [1]), which can be used repeatedly to split and recombine the wave packets with low loss. The beam splitter should provide an approximately unitary coupling between two quantum states, which is the necessary feature of a beam splitter for interactionfree measurements and many other quantum optics experiments [7,8]. The beam splitters used in electron interferometry experiments $[9,10]$ do not seem to fulfill these requirements. In particular, the electron biprism is a wavefront beam splitter and thus a different type of beam splitter than the one required. One possible setup that could achieve the desired coherent splitting and coupling of electron wavefunctions uses a microwave guide of electrons as a beam splitter [11]. It is based on a planar quadrupole guide which confines electrons transversally using microwave electric fields [12,13], similar to well-established techniques in ion traps [14]. Electron beam coherence studies on the microwave guide are currently underway.

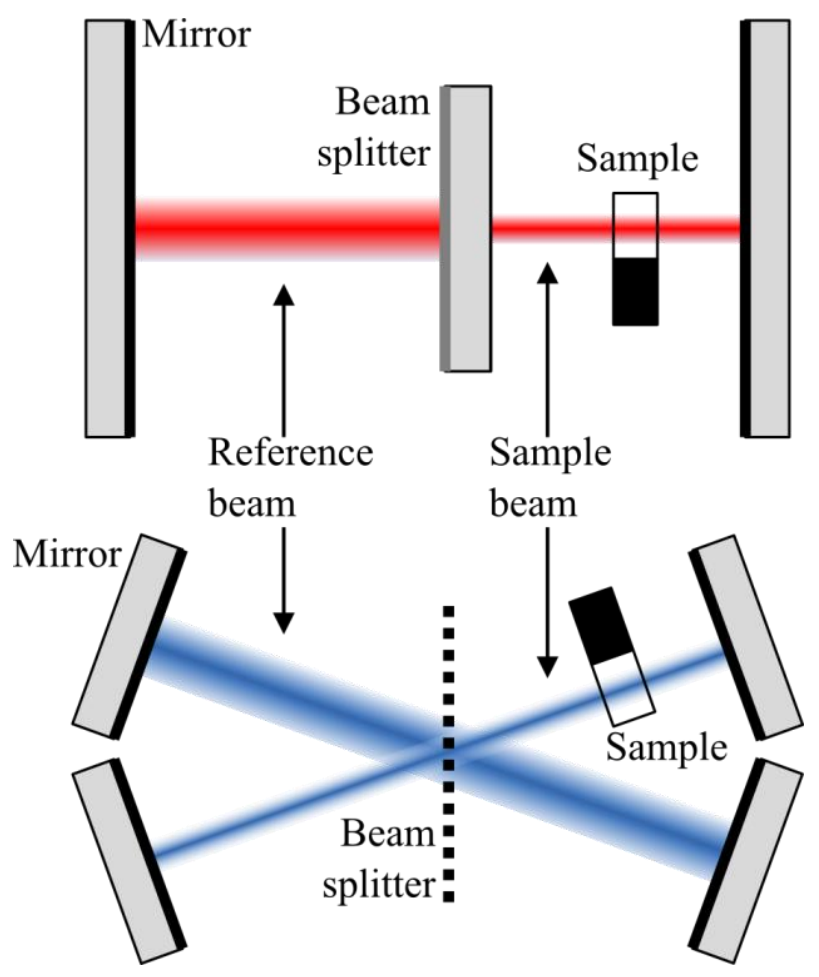

Figure 1. Sketch of an interaction-free measurement setup with photons and a possible realization of the quantum electron microscope proposal. The two setups are principally the same. A photon (upper diagram) or electron (lower diagram) beam is trapped in two cavities coupled by a beam splitter. The only major difference is the nature of the beam splitter, which is trivial for photons and could be based on diffraction for electrons. The beam starts out in the reference cavity and is transmitted to the sample cavity, where it interacts with a sample composed of transparent and opaque parts. If a transparent part is in the beam line, it does not affect the beam and it gets wholly transmitted after a certain time. If an opaque part is in the sample beam, however, it inhibits the transfer of intensity from the reference to the sample beam. By detecting the intensity in the reference beam after a certain time has passed, it is possible to find out whether the beam passes through an opaque or a transparent part of the sample. An image is obtained by raster-scanning the sample. 
While interaction-free measurements can distinguish between the presence and absence of an opaque object with arbitrarily low loss, the situation is more complicated if a semitransparent object is to be detected or its transparency is to be measured [4,15-17]. While detecting the presence or absence of a semitransparent sample is still possible with arbitrarily low damage (but requires a larger number of round trips $N$ than a detection of an opaque sample with the same loss probability) [15,17], there is a fundamental limit in quantum mechanical transparency measurements [16] which does not allow measuring the precise transparency of a sample with arbitrarily low damage. This shows that interactionfree measurements are still subject to quantum limits even if they violate classical intuition.

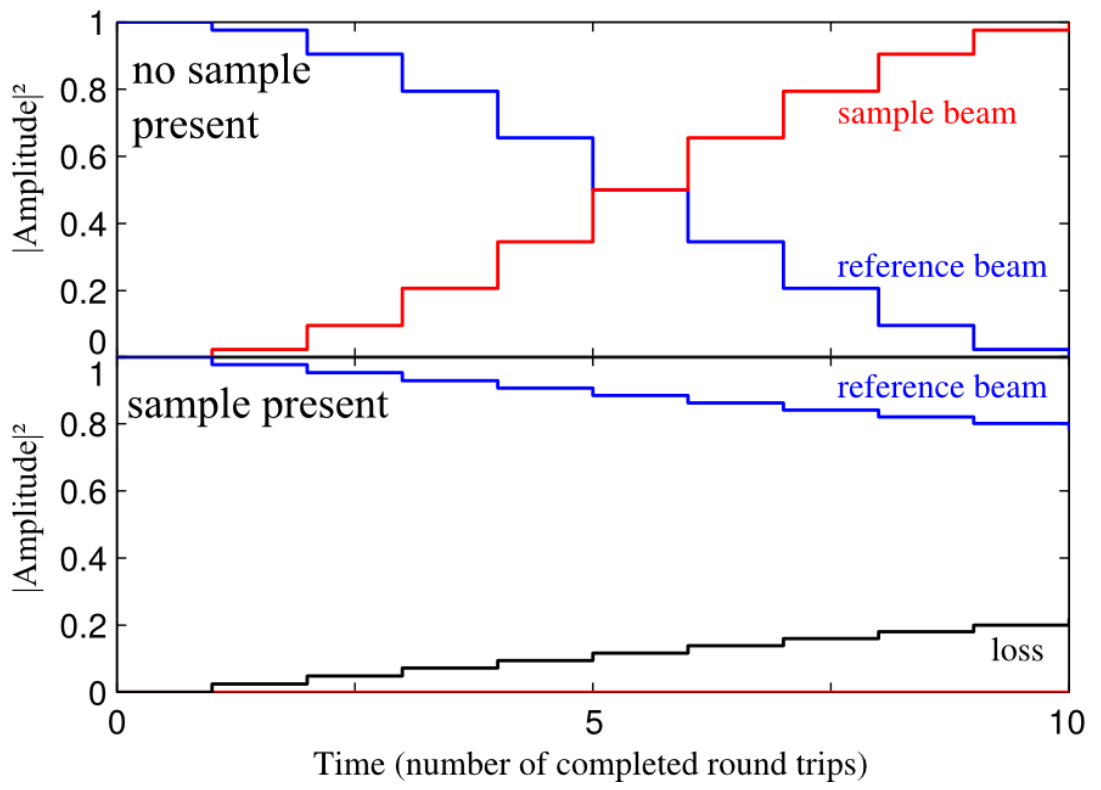

Figure 2. Behavior of the IFM particle as a function of time if there is no sample present and if there is a sample present. After every round trip, the probability $\left(=\mid\right.$ amplitude $\left.\left.\right|^{2}\right)$ of finding the particle in the different beams changes.

We have investigated the case of semitransparent samples and phase shifts in interaction-free measurements using numerical simulations [18]. One example simulation result for varying transparency (i.e., transmission probability of the probe particle through the sample) and $N=50$ is shown in Figure 3. For different transparencies, the probability of the particle being in any of the three states (reference beam, sample beam, lost) at the end of the IFM process varies continuously. For this reason, detecting only a single particle in either of the beams does not yield enough information to estimate the sample's transparency. Similar probability curves are found if the number of round trips $N$ is changed.

Interaction-free measurements can be used for measurements of transparency by running them repeatedly in order to estimate the probabilities and thus obtain the sample transparency. Such a repeated IFM obviously increases the average number of particles that are lost due to a sample interaction and, thus, the average radiation damage a sample receives in the measurement. Comparing the repeated IFM scheme to classical measurements of transparency (where one simply transmits particles through the sample and counts how many are lost), we find that IFMs do not allow a reduction of radiation damage in comparison to classical measurements. Interaction-free measurements and quantum electron 
microscopy therefore only outperform classical measurements when samples with a high contrast are to be distinguished (which is similar to the detection of the presence or absence of a sample), not in the precise measurement of transparency. More details can be found in Ref. [18].

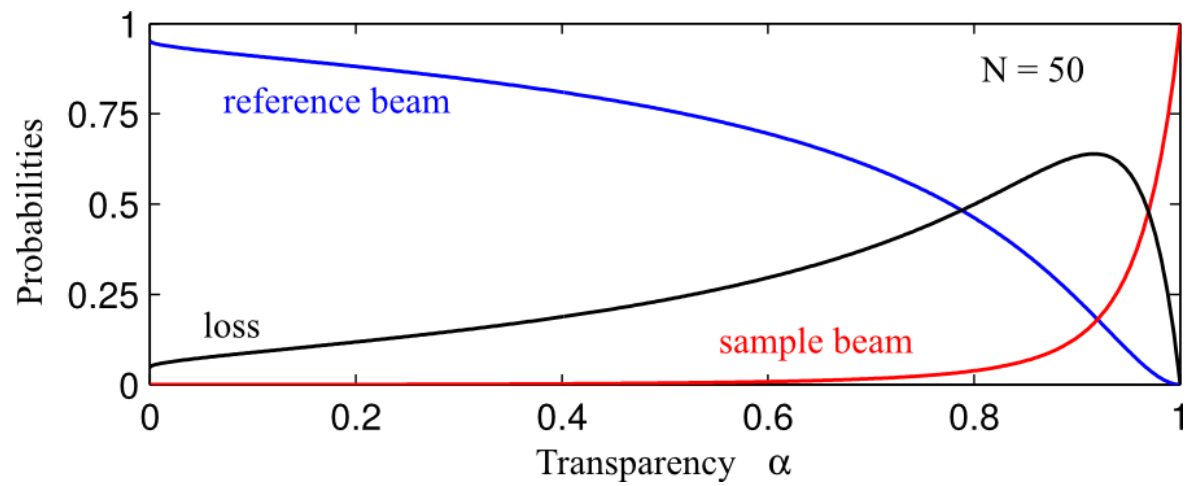

Figure 3. Probability of a particle's final state in an IFM with $N=50$ as a function of sample transparency. For different transparencies, the probability of losing the particle and of detecting it in the reference or sample beam changes.

To summarize, we discussed a novel quantum mechanical scheme of electron microscopy which will enable a significant reduction of radiation damage in imaging of high contrast samples. The major technical challenge in building such a device is the development of a suitable beam splitter. A promising candidate is based on a microwave chip [11]. We have also shown limits of the technique in transparency measurements. In conclusion, if the technical challenges in its realization can be overcome, quantum electron microscopy is a promising technique for certain tasks in imaging [19].

\section{References:}

[1] WP Putnam and MF Yanik, Phys. Rev. A 80 (2009), 040902.

[2] AC Elitzur and L Vaidman, Found. Phys. 23 (1993), p. 987.

[3] P Kwiat et al, Phys. Rev. Lett. 74 (1995), p. 4763.

[4] P Kwiat, Phys. Scripta 1998 (1998), p. 115.

[5] P Kwiat et al, Phys. Rev. Lett. 83 (1999), p. 4725.

[6] B Misra and ECG Sudarshan, J. Math. Phys. 18 (1977), p. 756.

[7] WP Schleich in "Quantum Optics in Phase Space", (Wiley, Berlin, 2005), chapter 13.

[8] A Zeilinger, Am. J. Phys. 49 (1981), p. 882.

[9] GF Missiroli, G Pozzi and U Valdrè, J. Phys. E. Sci. Instrum. 14 (1981), p. 649.

[10] F Hasselbach, Repts. Progr. Phys. 73 (2010), 016101.

[11] J Hammer et al, ArXiv preprint (2014), arxiv:1408.2658.

[12] J Hoffrogge et al, Phys. Rev. Lett. 106 (2011), 193001.

[13] J Hammer et al, Phys. Rev. Appl. 2 (2014), 044015.

[14] FG Major, VN Gheorge and G Werth in "Charged Particle Traps", (Springer, Berlin, 2005).

[15] G Mitchison and S Massar, Phys. Rev. A 63 (2001), 032105.

[16] G Mitchison, S Massar and S Pironio, Phys. Rev. A 65 (2002), 022110.

[17] H Azuma, Phys. Rev. A 74 (2006), 054301.

[18] S Thomas et al, Phys. Rev. A 90 (2014), 053840.

[19] The authors gratefully acknowledge funding from the Gordon and Betty Moore Foundation. 